\title{
The idiopathic hypereosinophilic syndrome
}

\author{
M A ALFAHAM, S D FERGUSON, B SIHRA, AND J DAVIES \\ Departments of Paediatrics and Cardiology, Royal Gwent Hospital, Newport, Gwent, Wales
}

SUMmARY A 14 year old girl with idiopathic hypereosinophilic syndrome is described. In addition to weight loss, anaemia, amenorrhoea, general lethargy, anorexia, mouth ulcers, blisters of hands and feet, and petechial skin rash, she had features of involvement of the cardiovascular system as the major complication. She responded well to treatment.

After a comprehensive search of the published reports 18 cases of this syndrome were identified in children under 16 years. Fifteen of these children had involvement of the cardiovascular system as the major source of their morbidity and mortality. Summary of the clinical details and laboratory, biopsy, and necropsy findings of the involvement of the various organ systems of the 18 children is presented.

Hypereosinophilia of over $1.5 \times 10^{9} / 1$ accompanies various diseases. In some cases no cause can be found and the hypereosinophilia may be accompanied by multiple organ involvement, especially of the cardiovascular system, with high morbidity and mortality. The idiopathic hypereosinophilic syndrome is primarily a disease of middle age, but an occasional case has been encountered in children.

\section{Case report}

A 14 year old white girl presented six weeks after an illness that lasted 10 days, in which she had developed mouth ulcers and blisters of the hands and feet. These had settled spontaneously, but the lethargy, nausea, and anorexia had persisted, and she had complained of aching limbs. She had described symptoms of increasing dyspnoea on minimal exertion and orthopnoea and developed a fine petechial rash two days before presentation. Her menses, which had started a year earlier, had stopped during the illness.

She had had asthma for two years, which was controlled with regular inhalations of beclomethasone and occasional inhalations of salbutamol. She was allergic to feathers. There was no recent infectious or relevant travel history.

On examination she was unwell, with a temperature of $38^{\circ} \mathrm{C}$, pulse rate 128 beats/minute, regular, respiratory rate of $28 /$ minute, blood pressure 130 / 100 , weight $46 \mathrm{~kg}$ (25th centile), and height $163 \mathrm{~cm}$ (60th centile). There was a sparse petechial rash on the upper trunk and upper arms, with splinter haemorrhages under her fingernails, and the pulps of her fingertips were tender on palpation. She was in congestive heart failure, with clinical evidence of a pericardial effusion. A grade $2 / 4$ pansystolic murmur radiated from the apex to the axilla. There was no lymphadenopathy or splenomegaly, but the liver was enlarged, $2 \mathrm{~cm}$ below the costal margin at the mid-clavicular line, and tender.

Investigations. Her haemoglobin concentration was $9.3 \mathrm{~g} / \mathrm{dl}$, mean corpuscular volume $78.7 \mathrm{fl}$, mean corpuscular haemoglobin $26.7 \mathrm{pg}$, platelets 315 $\times 10^{9} / 1$, white blood cells $13.4 \times 10^{9} / 1$ (neutrophils $45 \%$, lymphocytes $15 \%$, monocytes $1 \%$, and eosinophils $39 \%\left(5 \cdot 2 \times 10^{9} / 1\right)$, showing partial degranulation), and erythrocyte sedimentation rate $66 \mathrm{~mm}$ in the first hour. The prothrombin and kaolin cephalin clotting times were normal. Bone marrow biopsy and trephine examination showed a hypercellular marrow with pronounced eosinophilia but no evidence of malignancy. Chest $x$ ray film showed considerable cardiac enlargement, with a globular shaped heart, pulmonary oedema with vascular redistribution, and a small right sided pleural effusion. $\mathrm{M}$ mode and 2D echocardiography showed a large pericardial effusion and areas of increased echodensity in the region of the apex of the left ventricle and the posterior leaflet of the mitral valve, suggesting endocardial thickening. An electrocardiogram (ECG) showed sinus tachycardia.

The serum electrolytes, glucose, and liver function tests yielded normal results except for a low albumin concentration $(32 \mathrm{~g} / \mathrm{l})$ and slightly raised lactate dehydrogenase activity (351 IU/l (normal $100-300 \mathrm{IU} / \mathrm{l})$ ). Serum concentrations of vitamin $B_{12}$ 
were $501 \cdot 3 \mathrm{ng} / \mathrm{l}$ (normal $180-710 \mathrm{ng} / \mathrm{l}$ ), of folate were $2.2 \mu \mathrm{g} / \mathrm{l}$ ( normal $>2.5 \mu \mathrm{g} / \mathrm{l})$, and of ferritin were 70 $\mathrm{ng} / \mathrm{ml}$ (normal 10-64 ng/ml). Protein electrophoresis (in $\mathrm{g} / \mathrm{l}$ ) showed total protein 62, albumin 25, globulins 37, (alpha 4 (normal 2-4), alpha 10 (5-8.5), beta $7(7-11)$, and gamma $16(18-16)), \mathrm{IgG}$ $19 \cdot 22(7-19)$, IgM $1(0 \cdot 50-2 \cdot 2)$, and $\operatorname{IgA} 0.93(0 \cdot 9-$ $4 \cdot 5)$. Serum total IgE concentration was $154 \mathrm{KU} / \mathrm{l}$ (normal $<70 \cdot 3 \mathrm{KU} / \mathrm{l}$ ), functional $\mathrm{CH} 503.25 \mathrm{~min}$ $(1 \cdot 82-3 \cdot 62)$, C3 $209 \mathrm{mg} / \mathrm{dl}(70-160)$, C4 $29 \mathrm{mg} / \mathrm{dl}$ (10-30) properdin factor B $58 \cdot 1 \mathrm{mg} / \mathrm{dl}(20-50)$, and $C$ reactive protein $0.61 \mathrm{mg} / \mathrm{dl}(<0 \cdot 6)$. Rheumatoid factor was $240 \mathrm{IU} / \mathrm{ml}(<15)$. Antibodies for nuclear, mitochondrial, smooth muscle, parietal, canalicular, glomerular, and reticulin were negative. Antidouble stranded deoxyribonucleic acid (DNA) binding capacity was $0.9 \mathrm{mg} / \mathrm{l}$ (normal $<5 \mathrm{mg} / \mathrm{l}$ ).

Serial blood cultures and faeces examination, throat swab, Paul-Bunnell test, and tests for viral titres, hepatitis B surface antigen, and hepatitis A IgM yielded negative results. Antistreptolysin $\mathrm{O}$ was $<100$ Todd Units and antistreptococcal deoxyribonuclease B 1/75 (upper limit 1/200). Antibodies to cysticerci, filaria, fasciola, hydatid disease, toxocara, toxoplasma, Aspergillus fumigatus, Micropolyspora faeni, and Thermoactinomyces vulgaris were negative. Tests on urine samples showed protein $3+$, white blood cells $<1 /$ HPF, few granular casts and large number of epithelial cells and mucus, no growth, and a 24 hour protein excretion of $0.3 \mathrm{~g}$. Her karyotype was normal.

On the basis of the clinical syndrome, eosinophilia without a recognisable cause, and the echocardiogram findings, a diagnosis of idiopathic hypereosinophilic syndrome was made. She was begun on treatment with prednisolone $2 \mathrm{mg} / \mathrm{kg} /$ day, frusemide, and folic acid, and was anticoagulated with warfarin.

The signs of congestive heart failure improved appreciably within two days and the petechial rash disappeared within three days. The eosinophil count returned to normal within two days. The erythrocyte sedimentation rate, lactate dehydrogenase activity, rheumatoid factor, and serum IgE and folate concentrations returned to normal. The endocardial echoes and pericardial effusion decreased within a few days of beginning treatment and had completely disappeared within three months. Signs of mild mitral regurgitation confirmed by Doppler ultrasound persisted.

After her dramatic clinical improvement the steroids were gradually withdrawn and were stopped after six weeks. She then developed mild bronchospasm on excitement, which responded to inhaled salbutamol with concomitant improvement in the peak flow rate and forced expiratory volume in one second/forced vital capacity percentage, and was restarted on inhaled beclomethasone. Within two weeks of stopping oral steroids her eosinophil count increased to $1 \cdot 5 \times 10^{9} / 1$, and her total white blood cell count was $8.0 \times 10^{9} / 1$. She was restarted on prednisolone at a dose of $10 \mathrm{mg}$ on alternate days, after which the eosinophil count returned to normal. She remains anticoagulated with warfarin.

\section{Discussion}

Eosinophilia. Eosinophilia is the term applied to an increase in the total blood eosinophil concentrations. In normal individuals living in the United Kingdom the blood eosinophil count is less than $0.55 \times 10^{9} / 1$. The practice of referring to eosinophil concentrations as 'percentages' may be misleading and absolute figures $\left(\times 10^{9} / 1\right)$ should be used. Usually, the presence of eosinophilia has no detrimental effect on the body and there is evidence that it may have a role in the body defence mechanisms. It accompanies many disease states, mainly allergic and parasitic (Table 1), or it can be an incidental finding, but occasionally patients with eosinophilia are found to have one of the more serious and rarer causes, such as Hodgkin's disease. ${ }^{1}$ Treatment of the primary disease will usually resolve the eosinophilia.

Hypereosinophilia. Hypereosinophilia is the term applied empirically when the blood eosinophil count

Table 1 Some recognised causes of eosinophilia. All disorders mentioned might cause hypereosinophilia. Those that might be associated with a very high eosinophil count are underlined

1 Allergic disorders: asthma, hay fever, allergic rhinitis, urticaria, eczema pulmonary aspergillosis

2 Drug reactions

3 Parasitic infestations: visceral larva migrans (toxocariasis), trichinosis. ascariasis, hookworm disease, stronglyloidiasis, filariasis (tropical eosinophilia), schistosomiasis, hydatid disease, malaria

4 Skin disorders: eczema, dermatitis herpetiformis, exfoliative dermatitis, psoriasis, pemphigus

5 Infections: brucellosis, scarlet fever, acute infectious lymphocytosis, infections due to mycobacteria, chlamydia, cytomegalovirus, and Pneumocytis carinii

6 Haematological: Fanconi's anaemia, thrombocytopenia with absent radii. post-splenectomy

7 Neoplastic disorders: Hodgkin`s disease. lymphosarcoma, leukaemia. carcinoma of lung and gut

8 Connective tissue/vasculitis/granulomatous disorders, especially those involving the lungs: polyarteritis nodosa. Churg-Strauss syndrome. rheumatoid arthritis, systemic lupus erythematosis, scleroderma. cryptogenic pulmonary eosinophilia

9 Immune deficiency disorders: congenital immune deficiency syndromes. hyper-IgE with infections

10 Miscellaneous: liver cirrhosis, radiation treatment. Crohn's disease ulcerative colitis, peritoneal dialysis, congenital heart disease, familial eosinophilia (? autosomal dominant), idiopathic hypereosinophilic syndrome (including eosinophilic leukaemia) 
is $1.5 \times 10^{9} / 1$ or higher. Some disorders usually give rise to counts above $1.5 \times 10^{9} / 1,{ }^{1}$ - for example, drug reactions, intrinsic asthma (a surprising fact compared with patients with extrinsic asthma, in whom the eosinophil count is usually below $1 \times 10^{9} / 1$ ), parasitic diseases, vasculitis and granulomatous diseases, and neoplasms. Certain other diseases are associated with striking eosinophilia: leucocyte counts of $30 \times 10^{9} / 1-100 \times 10^{9} / 1$ or higher may be seen where $50-90 \%$ of the blood leucocytes may be eosinophils. Diseases that are more commonly associated with such high counts are underlined ${ }^{2}$ in Table 1, but in general any of the disease processes mentioned in the Table - that is, any cause of eosinophilia-may be associated with hypereosinophilia of whatever degree.

Hypereosinophilia can be primary. This may be an isolated finding. On the other hand, it might be associated with organ system dysfunction of varying extent and severity, the cardiovascular and nervous system and skin being most commonly involvedthe idiopathic hypereosinophilic syndrome.

The idiopathic hypereosinophilic syndrome. In 1968 Hardy and Anderson drew attention to the fact that persistent hypereosinophilia of any type could be associated with a range of similar complications and they grouped these together as the hypereosinophilic syndrome. ${ }^{3}$ Chusid et al confirmed this in 1975 but restricted the diagnosis of hypereosinophilic syndrome to patients in whom no underlying cause for the hypereosinophilia could be shown, ${ }^{4}$ so that the term idiopathic hypereosinophilic syndrome is applied when the following criteria are fulfilled:

(1) Eosinophilia $\geqslant 1.5 \times 10^{9} / 1$;

(2) Persists for at least six months or fatal in a shorter time;

(3) Results in organ system dysfunction;

(4) Absence of a recognised cause for the eosinophilia.

The term encompasses various diagnostic terms applied previously to a range of cases with almost similar natural history, physical findings, laboratory data, and autopsy findings, including eosinophilic leukaemia, disseminated eosinophilic collagen disease, and Loeffler's fibroplastic endocarditis with eosinophilia. The condition called eosinophilic gastroenteritis is probably part of the wide range of this syndrome. ${ }^{5}$

The idiopathic hypereosinophilic syndrome has a wide range of severity with at least three different forms. Some patients only have hypereosinophilia with lung involvement and angio-oedema. Others present with (or develop) severe cardiac and central nervous system complications. A third group have eosinophil cytogenic abnormalities and other fea- tures of a leukaemic disease, characterised as eosinophilic leukaemia. Occasionally, the second form terminates as a blast cell leukaemia. ${ }^{6}$ Hypereosinophilia as an isolated finding is not considered to be a subgroup of this syndrome and probably has no special clinical relevance, although the patient will require regular reviews to exclude an occult underlying disease process.

\section{Aetiology}

Several theories have been put forward to explain the idiopathic hypereosinophilic syndrome. Some authors have claimed that it is an autoimmune disease. ${ }^{7}$ It is also possibly of a neoplastic nature. ${ }^{8}$ Parrillo et al showed a variety of immunological abnormalities in the patients they studied. ${ }^{9}$ In most of the cases described by Spry et al the findings suggested that a parasitic or allergic disease had preceded the onset of hypereosinophilia, and some had high serum IgE concentrations. ${ }^{10}$ These findings raised the possibility that an initial eosinopoietic stimulus may lead to a late and exaggerated response, which persists. Carey et al reported a case of transient hypereosinophilia in an infant of a mother with this syndrome and suggested that the infant's hypereosinophilia could be a response to an eosinophilia producing factor by the mother, with transplacental transfer. ${ }^{11}$

A definitive cause for this syndrome, however, has not been identified, but perhaps understanding the factors that influence the production and distribution of the eosinophil and the mechanisms of tissue damage might give some insight into its aetiology.

Factors that influence blood and tissue eosinophil concentration. Eosinophils normally constitute a small proportion of the circulating leucocytes. A diurnal variaton that is the converse of the variation in plasma corticosteroid concentrations occurs, with blood eosinophilia peaking at night. The production of the eosinophil in the bone marrow is under the control of the $\mathrm{T}$ lymphocytes. Lymphocytes also produce factors that are chemotactic for eosinophils. Once eosinophils are released from the marrow they circulate in the blood only briefly, with a half life of six to 12 hours. They leave the blood stream and are distributed in tissues where they persist for at least several days, being a tissue dwelling cell. They are most abundant in those tissues with epithelial surfaces exposed to the environment, including the gastrointestinal tract, the skin, and the upper respiratory tract. $^{12}$

Apart from the lymphocytes, several other factors may influence eosinophil concentrations in blood and tissue. On triggering and degranulating the mast 
604 Alfaham, Ferguson, Sihra, and Davies

Table 2 Summary of clinical, biopsy, and necropsy findings of 19 children with the idiopathic hypereosinophilic syndrome

\begin{tabular}{|c|c|c|c|c|c|c|c|c|c|c|}
\hline Author (years) & $\begin{array}{l}\text { Age at } \\
\text { onset } \\
\text { (years) }\end{array}$ & Sex & Presenting symptoms & $\begin{array}{l}\text { Cardiovascular } \\
\text { system }\end{array}$ & Nervous system & Skin/muscle joints & Renal & Pulmonary & Gastrointestinal tract & Hepatic \\
\hline Bentley $(1961)^{15}$ & 5 & $\mathbf{M}$ & $\begin{array}{l}\text { Fever, macular rash, } \\
\text { petechial rash, coma }\end{array}$ & $\begin{array}{l}\text { Postmortem examina- } \\
\text { tion: Myocardial } \\
\text { petechiac and scar- } \\
\text { ring. mural thrombus } \\
\text { in left ventricle }\end{array}$ & $\begin{array}{l}\text { Coma, decorticate. } \\
\text { posture. Cerebrospi- } \\
\text { nal fluid: No abnor- } \\
\text { mality. Postmortem } \\
\text { examination: Myclo- } \\
\text { blastic infiltratc of } \\
\text { meninges and brain }\end{array}$ & $\begin{array}{l}\text { Macular and petce- } \\
\text { hial rash. Sub- } \\
\text { cutancous nodules } \\
\text { over parictal bonses. } \\
\text { Biopsy: Lymphoid } \\
\text { and eosinophil infil- } \\
\text { tration }\end{array}$ & $\begin{array}{l}\text { Posimortem ex- } \\
\text { amination: Petechiac } \\
\text { in both kidncys. } \\
\text { Blast cellls infiltration } \\
\text { of kidncys and } \\
\text { bladder }\end{array}$ & $\begin{array}{l}\text { Dry cough. Chest } x \\
\text { ray: Lung infiltrates. } \\
\text { Postmortem ex- } \\
\text { amination: Hacmor- } \\
\text { rhagic arcas. Pul- } \\
\text { monary ocdcma and } \\
\text { mycloblast infiltra- } \\
\text { tion }\end{array}$ & $\begin{array}{l}\text { Postmontem ex- } \\
\text { amination: Stomach } \\
\text { dilatation. Pctcchiac } \\
\text { all over gastrointes- } \\
\text { tinal tract }\end{array}$ & $\begin{array}{l}\text { Hepatomcgaly. Post- } \\
\text { mortem examination: } \\
\text { Blast cells infiltration }\end{array}$ \\
\hline Chusid $(1975)^{4}$ & 5.5 & $\mathbf{M}$ & $\begin{array}{l}\text { Periodic attacks of } \\
\text { fever, rash. di- } \\
\text { arrhoea. and angio- } \\
\text { ocdema }\end{array}$ & Nonc & $\begin{array}{l}\text { Abnormal clcc- } \\
\text { troencephalogram }\end{array}$ & $\begin{array}{l}\text { Rash, angio-ocdema. } \\
\text { Skin biopsy: Pcrivas- } \\
\text { culitis, cosinophil in- } \\
\text { filtration }\end{array}$ & $\begin{array}{l}\text { Mild to moderate in- } \\
\text { termittent proteinur- } \\
\text { ia }\end{array}$ & Transicnt infiltration & None & $\begin{array}{l}\text { Biopsy: 'Eosinophilic } \\
\text { triaditis' }\end{array}$ \\
\hline De Vaan $(1979)^{16}$ & 1 & $\mathbf{F}$ & $\begin{array}{l}\text { Refusing to walk or } \\
\text { stand }\end{array}$ & $\begin{array}{l}\text { Gallop rhythm and } \\
\text { sinus tachycardia. } \\
\text { Postmorrem examina- } \\
\text { tion: Endocardial } \\
\text { thickening of left } \\
\text { atrium and subcn- } \\
\text { docardial fibrosis with } \\
\text { iron pigment dcposi- } \\
\text { tion in myocardium } \\
\text { of both ventricles and } \\
\text { left atrium. Coronary } \\
\text { thrombosis. Eosi- } \\
\text { nophil infiltration. in- } \\
\text { cluding blast cells in } \\
\text { myocardium }\end{array}$ & None & $\begin{array}{l}\text { Refusing to walk or } \\
\text { stand. Skin hiopsy: } \\
\text { Papillomatosis and } \\
\text { scattered maturc } \\
\text { cosinophil infiltra- } \\
\text { tion and hyperker- } \\
\text { atosis. Muscle } \\
\text { biopsy: Somc focal } \\
\text { atrophy, no cosi- } \\
\text { nophil infiltration }\end{array}$ & $\begin{array}{l}\text { Postmortem ex- } \\
\text { amination: Eosi- } \\
\text { nophil infiltration, } \\
\text { including blast cells }\end{array}$ & $\begin{array}{l}\text { Pncumonia. Post- } \\
\text { mortem examination: } \\
\text { Increase in wcight of } \\
\text { lungs. Vascular } \\
\text { thrombi. Areas of } \\
\text { red blood cell cxtra- } \\
\text { vasation, fibrin in } \\
\text { alvelar spaces, and } \\
\text { formation of hyaline } \\
\text { membrancs. Mac- } \\
\text { rophages and cosi- } \\
\text { nophils in alveolar } \\
\text { spaces }\end{array}$ & None & $\begin{array}{l}\text { Hepatomegaly. Biop. } \\
\text { Diffuse eosinophil in } \\
\text { tration. Postmortem } \\
\text { amination: Enlarged. } \\
\text { eosinophil infiltration } \\
\text { including blast cells }\end{array}$ \\
\hline Donohuc (1950) $)^{17}$ & 7 & $\mathbf{M}$ & Joint pains and fever & $\begin{array}{l}\text { Congestive heart fai- } \\
\text { lure, cardiomegaly, } \\
\text { mild hypertension, } \\
\text { systolic murmur, mit- } \\
\text { ral regurgitation }\end{array}$ & None & $\begin{array}{l}\text { Subcutancous } \\
\text { ocdema. } \\
\text { arthralgia }\end{array}$ & Nonc & $\begin{array}{l}\text { Bilatcral basal } \\
\text { crepitations }\end{array}$ & None & Hepatomegaly \\
\hline Engback $(1942)^{18}$ & 7 & $\mathbf{M}$ & $\begin{array}{l}\text { Headaches, fever. } \\
\text { neck stiffness, and } \\
\text { arthralgia }\end{array}$ & $\begin{array}{l}\text { Congestive heart fai- } \\
\text { lure, cardiomegaly. } \\
\text { Posimortem examina- } \\
\text { tion: Hypertrophy. } \\
\text { dilatation and parietal } \\
\text { thrombus in both ven- } \\
\text { tricles and myocardial } \\
\text { scarring/obliteration } \\
\text { of coronaries }\end{array}$ & Not described & Not described & Not described & $\begin{array}{l}\text { Posimontem ex- } \\
\text { amination: Lungs in- } \\
\text { durated. cyanotic. } \\
\text { mottled. and in- } \\
\text { flamed. Thrombi. } \\
\text { eedema, and eosi- } \\
\text { nophil infiltration }\end{array}$ & Not described & Nonc \\
\hline $\begin{array}{l}\text { Engfeldt (1956): } \\
\text { Case } 1\end{array}$ & 8 & $\mathbf{M}$ & $\begin{array}{l}\text { Fatigue, anorexia. } \\
\text { abdominal pain. } \\
\text { vomiting, dyspnoca. } \\
\text { cough, pains in back } \\
\text { and neck }\end{array}$ & $\begin{array}{l}\text { Terminal acute heart } \\
\text { failure. Postmortem } \\
\text { examination: Biven- } \\
\text { tricular hypertrophy, } \\
\text { thrombus in right au- } \\
\text { ricie and ventricles. } \\
\text { Endo-, myo-, and } \\
\text { pericardial scarring } \\
\text { and inflammatory } \\
\text { cells infiltratc, mainly } \\
\text { cosinophils }\end{array}$ & $\begin{array}{l}\text { Electroencephalo- } \\
\text { gram: Dysrhythmic }\end{array}$ & $\begin{array}{l}\text { Pains in back and } \\
\text { neck. Postmortem ex- } \\
\text { amination: Eosi- } \\
\text { nophil infiltration of } \\
\text { musclc }\end{array}$ & $\begin{array}{l}\text { Moderate albuminur- } \\
\text { ia, hyaline casts, and } \\
\text { red blood cclls in } \\
\text { urine. Uraemia. } \\
\text { Postmortem ex- } \\
\text { amination: Eosi- } \\
\text { nophil infiltration of } \\
\text { kidneys }\end{array}$ & $\begin{array}{l}\text { Dyspnoca, cough. } \\
\text { Chest } x \text { ray: Peri- } \\
\text { bronchial thickening } \\
\text { and pleural effusion. } \\
\text { Postmortem ex- } \\
\text { amination: Eosi- } \\
\text { nophil infiltrate }\end{array}$ & $\begin{array}{l}\text { Abdominal pain and } \\
\text { vomiting }\end{array}$ & $\begin{array}{l}\text { Hepatomegaly. Post } \\
\text { mortem examination } \\
\text { Eosinophil infiltratio }\end{array}$ \\
\hline Case 2 & 10 & $\mathbf{M}$ & $\begin{array}{l}\text { Headache, fcver. } \\
\text { rash. hyperkeratosis. } \\
\text { and scaling of facc. } \\
\text { arms, and legs, pro- } \\
\text { nounced alopecia. } \\
\text { conjunctivitis, myal- } \\
\text { gia }\end{array}$ & $\begin{array}{l}\text { Cardiomegaly, apical } \\
\text { systolic murmur }\end{array}$ & None & $\begin{array}{l}\text { See presenting symp- } \\
\text { toms. Subcutaneous } \\
\text { oedema and redness. } \\
\text { over wrists and } \\
\text { knees. Arthralgia. } \\
\text { Biopsy of skin and } \\
\text { muscle: Inflamma- } \\
\text { tory cells. especially } \\
\text { cosinophils and } \\
\text { plasma cells. Non- } \\
\text { specific changes in } \\
\text { small blood vessels. } \\
\text { thrombosis, and en- } \\
\text { darteritic changes }\end{array}$ & Slight proteinuria & $\begin{array}{l}\text { Atelectasis of right } \\
\text { middle lobe. Pleural } \\
\text { effusion }\end{array}$ & None & Hepatomegaly \\
\hline Foasso $(1984)^{19}$ & 6.7 & $\mathbf{F}$ & $\begin{array}{l}\text { Sore throat. Tubercu- } \\
\text { losis over previous } 12 \\
\text { months: on isoniazid. } \\
\text { rifampicin, etham- } \\
\text { butal }\end{array}$ & $\begin{array}{l}\text { Congestive heart fai- } \\
\text { lure. cardiomegaly, } \\
\text { hypertension. Echo- } \\
\text { cardiogram: Dilated } \\
\text { left ventricle and left } \\
\text { auricle. Catheserisa- } \\
\text { tion: Left ventricular } \\
\text { lumen was reduced }\end{array}$ & Not described & $\begin{array}{l}\text { Ulccration of finger } \\
\text { tips. Swelling of right } \\
\text { sternocleidomastoid. } \\
\text { Skin biopsy: Peri- } \\
\text { vascular inflamma- } \\
\text { tory cell infiltration. } \\
\text { Muscle biopsy: En- } \\
\text { dothelial thickening } \\
\text { and alteration of } \\
\text { media, no perivascu- } \\
\text { lar inflammation }\end{array}$ & $\begin{array}{l}\text { Microscopic haema- } \\
\text { turia. Biopsy: Focal } \\
\text { glomerulonephritis }\end{array}$ & $\begin{array}{l}\text { Pulmonary hyperten- } \\
\text { sion and ocdema }\end{array}$ & Not described & Hepatomegaly \\
\hline
\end{tabular}


The idiopathic hypereosinophilic syndrome 605 क

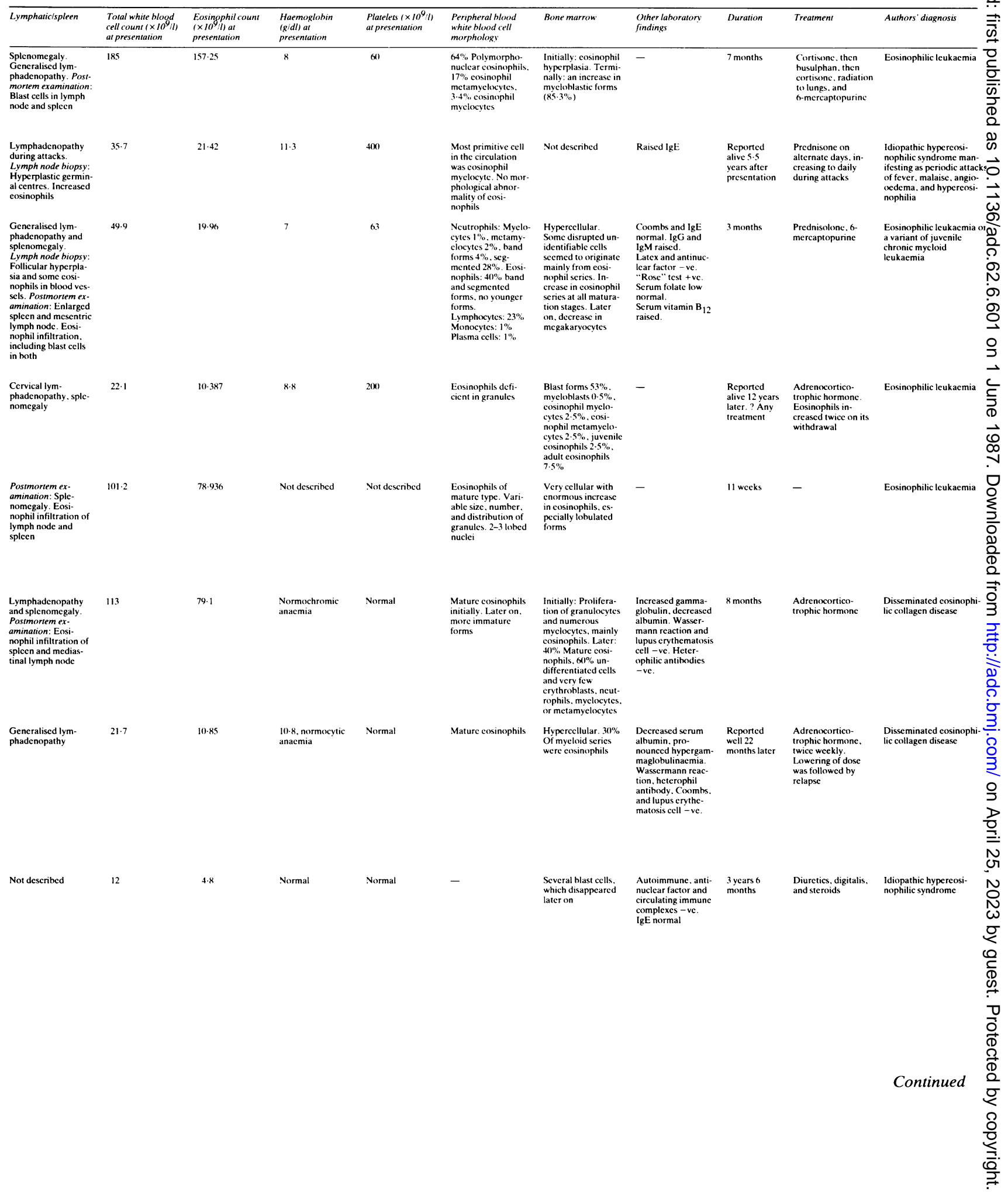


606 Alfaham, Ferguson, Sihra, and Davies

Table 2 Summary of clinical, biopsy, and necropsy findings of 19 children with the idiopathic hypereosinophilic syndrome (continued)

\begin{tabular}{|c|c|c|c|c|c|c|c|c|c|c|}
\hline Author (years) & $\begin{array}{l}\text { Age at } \\
\text { onset } \\
\text { (years) }\end{array}$ & Sex & Presenting symptoms & $\begin{array}{l}\text { Cardiovascular } \\
\text { system }\end{array}$ & Nervous system & Skin/muscle joints & Renal & Pulmonary & Gastrointestinal tract & Hepatic \\
\hline Goldstcin $(1952)^{20}$ & 7 & $\mathbf{M}$ & $\begin{array}{l}\text { Fever. Pain in abdo- } \\
\text { men and right axilla }\end{array}$ & Nonc & Nonc & Nonc & Nonc & $\begin{array}{l}\text { Nonc. Chest } x \text { ray: } \\
\text { No abnormality }\end{array}$ & None & None \\
\hline
\end{tabular}

\begin{tabular}{|c|c|c|c|c|c|c|c|c|c|c|}
\hline $\begin{array}{l}\text { Herrera-Goepfert } \\
(1985)^{21}\end{array}$ & 9 & $\mathbf{M}$ & $\begin{array}{l}\text { Myalgia, arthralgia, } \\
\text { fever, delirium, facial } \\
\text { and leg ocdema. } \\
\text { progressive dyspnoea }\end{array}$ & $\begin{array}{l}\text { Congestivc heart fai- } \\
\text { lure, cardiomegaly. } \\
\text { systolic murmur (mit- } \\
\text { ral regurgitation). } \\
\text { Postmortem examina- } \\
\text { tion: Ventricular } \\
\text { dilatation and hyper- } \\
\text { trophy. Thrombi in } \\
\text { auricle and right ven- } \\
\text { triclc. Extensive sub- } \\
\text { cndocardial fibrosis. } \\
\text { Thrombosis and in- } \\
\text { flammatory cell infil- } \\
\text { tration by lympho- } \\
\text { cytcs, plasma cells. } \\
\text { and cosinophils in } \\
\text { blod vessels of } \\
\text { myocardium }\end{array}$ & $\begin{array}{l}\text { Nonc. Postmortem } \\
\text { examination: Cyster- } \\
\text { icus in left putamen, } \\
\text { thought to be an in- } \\
\text { cidental finding }\end{array}$ & $\begin{array}{l}\text { Myalgia, arthralgia. } \\
\text { Muscle biopsy: Non- } \\
\text { specific }\end{array}$ & Not described & $\begin{array}{l}\text { Chest } x \text { ray: Infiltra- } \\
\text { tive pulmonary } \\
\text { changes. Postmortem } \\
\text { examination: Throm- } \\
\text { bi, infarcts, and in- } \\
\text { flammatory cclls in- } \\
\text { filtration by lympho- } \\
\text { cytes, plasma cells, } \\
\text { and cosinophils in } \\
\text { blood vessels of lungs }\end{array}$ & Not described & $\begin{array}{l}\text { Hepatomegaly. Post- } \\
\text { mortem examination: } \\
\text { Eosinophil infiltration }\end{array}$ \\
\hline Knorr $(1958)^{22}$ & 7.5 & $\mathbf{F}$ & $\begin{array}{l}\text { Fever, sore throat, } \\
\text { pains in fect, anor- } \\
\text { exia, irritability }\end{array}$ & $\begin{array}{l}\text { Acutc heart failure. } \\
\text { Cardiomcgaly, systo- } \\
\text { lic murmur (mitral } \\
\text { regurgitation). Post- } \\
\text { morlem examination: } \\
\text { Left atrium greatly di- } \\
\text { lated. Ventricles en- } \\
\text { larged, subendo- and } \\
\text { myocardial fibrosis. } \\
\text { cspecially of left. En- } \\
\text { docardial fibrosis, im- } \\
\text { mediately under the } \\
\text { postcrior leaflet of } \\
\text { mitral valve, extend- } \\
\text { ing into chordec ten- } \\
\text { dinii. Destruction of } \\
\text { postcrior reaflet of } \\
\text { mitral valve. Arterial } \\
\text { cmbolus in right leg }\end{array}$ & $\begin{array}{l}\text { Extensive involuntary } \\
\text { chorciform hyper- } \\
\text { kincsia, pronounced } \\
\text { hypotonia, absent } \\
\text { abdominal reflexes }\end{array}$ & $\begin{array}{l}\text { Pains in fect. Gener- } \\
\text { alised coarse brown' } \\
\text { macular rash. } \\
\text { Erythema annulare. } \\
\text { Joints normal }\end{array}$ & $\begin{array}{l}\text { Urine: Numcrous } \\
\text { cpithelial cells. no } \\
\text { other abnormality }\end{array}$ & $\begin{array}{l}\text { Attacks of broncho- } \\
\text { pneumonia. dysp- } \\
\text { neea and wheczing. } \\
\text { associated with car- } \\
\text { diac decompensa- } \\
\text { tion. Posmortem ex- } \\
\text { amination: Lung } \\
\text { ocdema. Pncumonic } \\
\text { changes with eosi- } \\
\text { nophil infiltration }\end{array}$ & None & 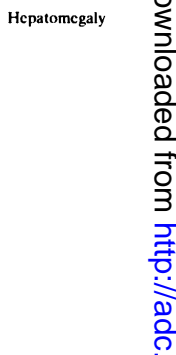 \\
\hline Libanoff $(1976)^{23}$ & 9 & M & $\begin{array}{l}\text { Dizzy spells, fatiguc, } \\
\text { shorness of breath }\end{array}$ & $\begin{array}{l}\text { Congestive heart fai- } \\
\text { lurc. cardiomegaly. } \\
\text { systolic murmur (mit- } \\
\text { ral regurgitation). } \\
\text { Postmortem examina- } \\
\text { tion: Extensive cn- } \\
\text { docardial and } \\
\text { myocardial fibrosis in } \\
\text { both ventricles, most } \\
\text { severe at the apical } \\
\text { position of right ven- } \\
\text { tricle. Anterior leaflet } \\
\text { of mitral value was } \\
\text { destroyed. Pericardial } \\
\text { cffusion. No cosi- } \\
\text { nophil infiltratc }\end{array}$ & None & None & None & $\begin{array}{l}\text { Dyspnoca. Chest } x \\
\text { ray: Increased vascu- } \\
\text { lar markings. Post- } \\
\text { mortem exammination: } \\
\text { Plcural cffusion }\end{array}$ & $\begin{array}{l}\text { Postmortem } \\
\text { examination: } \\
\text { Ascites }\end{array}$ & $\begin{array}{l}\text { Hepatomcgaly. Post } \\
\text { mortem examination } \\
\text { Liver congestion }\end{array}$ \\
\hline Olson (1982) & 9 & $\mathbf{M}$ & $\begin{array}{l}\text { Fatiguc, anorcxia. } \\
\text { weight loss. fever. } \\
\text { cough }\end{array}$ & $\begin{array}{l}\text { Congestive hcart fai- } \\
\text { lure. cardiomegaly, } \\
\text { systolic murmur (mit- } \\
\text { ral regurgitation). } \\
\text { Postmortem examina- } \\
\text { tion: Biventricular cn- } \\
\text { domyocardial fibrosis } \\
\text { (mural thrombi in left } \\
\text { ventricle) }\end{array}$ & Not described & $\begin{array}{l}\text { Skeletal muscle } \\
\text { biopsy: Normal }\end{array}$ & Not described & $\begin{array}{l}\text { Cough. Chest x ray: } \\
\text { Bilatcral alvcolar } \\
\text { pncumonitis. Biopsy: } \\
\text { Hypersensitivity } \\
\text { pneumonitis }\end{array}$ & Not described & $\begin{array}{l}\text { Clinically not describqu } \\
\text { Postmortem examina- } \\
\text { tion: Chronic passive } \\
\text { congestion and centrie } \\
\text { lobular necrosis }\end{array}$ \\
\hline Rasche $(1973)^{25}$ & 5 & $\mathbf{F}$ & $\begin{array}{l}\text { Abdominal pain, di- } \\
\text { arrhoca, vomiting. } \\
\text { fever }\end{array}$ & $\begin{array}{l}\text { Congestivc hcart fai- } \\
\text { lurc. cardiomegaly. } \\
\text { Postmortem examina- } \\
\text { tion: Biventricular } \\
\text { dilatation, thrombi in } \\
\text { both ventricles and } \\
\text { right atrium, contain- } \\
\text { ing abundant cosi- } \\
\text { nophils. Coronary } \\
\text { thrombrosis and } \\
\text { myocardial necrosis }\end{array}$ & $\begin{array}{l}\text { Irritability. confu- } \\
\text { sion. restlessness. } \\
\text { coma. Postmortem } \\
\text { examination: Throm- } \\
\text { bi in crebral arter- } \\
\text { ioles and venulcs. } \\
\text { nccrosis in thalamus } \\
\text { and cerchcllum }\end{array}$ & None & $\begin{array}{l}\text { Normal scrum crcati- } \\
\text { nine and urea. Post- } \\
\text { mortem examination: } \\
\text { Focal proliferative } \\
\text { glomerulitis }\end{array}$ & $\begin{array}{l}\text { Dyspnoca. Chest } x \\
\text { ray: Lincar opacitics } \\
\text { in both bases. Post- } \\
\text { mortem examination: } \\
\text { Red and congested. } \\
\text { Vascular thrombosis } \\
\text { and lung infarct }\end{array}$ & $\begin{array}{l}\text { Abdominal pain. } \\
\text { diarrhoca, and } \\
\text { vomiting }\end{array}$ & $\begin{array}{l}\text { Hepatomcgaly. Pro- } \\
\text { onged prothombin } \\
\text { time. Postmortem ex - } \\
\text { amination: Thrombi in } \\
\text { sinusoids and cosinopmint } \\
\text { infiltration } \\
\text { Continued }\end{array}$ \\
\hline
\end{tabular}


The idiopathic hypereosinophilic syndrome 607

\begin{tabular}{|c|c|c|c|c|c|c|c|c|c|c|}
\hline Lymphatic/spleen & $\begin{array}{l}\text { Total white blogd } \\
\text { cell count }\left(\times 10^{9}\right) \\
\text { cat presentation }\end{array}$ & $\begin{array}{l}\text { Eosingphil count } \\
\left(\times 10^{9 / 1) \text { at }}\right. \\
\text { presentation }\end{array}$ & $\begin{array}{l}\text { Haemoglobin } \\
\text { (g/dl) at } \\
\text { presentation }\end{array}$ & $\begin{array}{l}\text { Platelets }\left(\times 11^{9}(1)\right. \\
\text { at presentation }\end{array}$ & $\begin{array}{l}\text { Peripheral blood } \\
\text { whire blood cell } \\
\text { morphology }\end{array}$ & Bone marrow & $\begin{array}{l}\text { Other laboratory } \\
\text { findings }\end{array}$ & Duration & Treatment & Authors' diagnosis \\
\hline $\begin{array}{l}\text { Splenomegaly. } \\
\text { Generalised lym- } \\
\text { phadenopathy. Ten- } \\
\text { der lymph node in } \\
\text { right axilla. Lymph } \\
\text { nodebiopsyy E Eosi- } \\
\text { nophil and poly- } \\
\text { morph infiltration. } \\
\text { Fibrosis. In general. } \\
\text { picture is of lym- } \\
\text { phadenitis., no other } \\
\text { specific changes. no } \\
\text { parasites and no cal- } \\
\text { cification }\end{array}$ & 38.3 & 19.9 & $\begin{array}{l}\text { Moderate } \\
\text { anacmia" }\end{array}$ & Not described & $\begin{array}{l}\text { Neutrophil: band } \\
\text { forms } 3 \% \text {. seg- } \\
\text { mented forms } 31 \% \text {. } \\
\text { Eosinophil scg- } \\
\text { mented forms } 52 \% \text {. } \\
\text { Lyymphocytec } 13 \% \text {. } \\
\text { Monocytes } 1 \% \text {. } \\
\text { Normal granulcs in } \\
\text { cosinophils }\end{array}$ & $\begin{array}{l}\text { Pronounced in- } \\
\text { creasc in cosi- } \\
\text { nophist. Otherwise } \\
\text { normal }\end{array}$ & $\begin{array}{l}\text { Wassermann reac- } \\
\text { tion-vc. } \\
\text { Mantoux test } 1 / \\
\text { 1000 +ve. } \\
\text { Globulin and albu- } \\
\text { min normal }\end{array}$ & 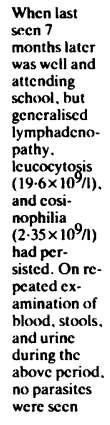 & $\begin{array}{l}\text { Nonc. The fever. } \\
\text { splenomegaly. and } \\
\text { anacmia dis- } \\
\text { appeared spon- } \\
\text { taneously }\end{array}$ & Eosinophilia infectiosa \\
\hline $\begin{array}{l}\text { Generaliscd lym- } \\
\text { phadenopathy. } \\
\text { Lymph node biopsy: } \\
\text { Non-specific. Post- } \\
\text { mortem examination: } \\
\text { Splecn and lymph } \\
\text { nodc cnlarged. Eosi- } \\
\text { nophil infiltration }\end{array}$ & Not specified & Not specificed & Not described & Not described & $\begin{array}{l}\text { At some stage whitc } \\
\text { blood gcll count was } \\
85 \times 10^{\prime} / \text {, with } 75 \% \\
\text { cosinophils of nor- } \\
\text { mal morphology }\end{array}$ & $\begin{array}{l}\text { Increased cosi- } \\
\text { nophils of normal } \\
\text { morphology. Post- } \\
\text { mortem examina- } \\
\text { tion: IIcrascd cosi- } \\
\text { nophils }(98 \%)\end{array}$ & $\begin{array}{l}\text { Total serum protein } \\
82 \mathrm{~g} / 100 \mathrm{ml} \text {. inver- } \\
\text { sion of albumin/ } \\
\text { globulin ratio. } \\
\text { Latex test, lupus } \\
\text { crythematosis cells } \\
\text { and antinuclcar } \\
\text { factor }-\mathrm{ve}\end{array}$ & 6 months & Not described & $\begin{array}{l}\text { Idiopathic hypercosi- } \\
\text { nophilic syndrome }\end{array}$ \\
\hline
\end{tabular}

Generalised lymphadenopathy. Splc-

$18 \cdot 3$

Hypochromic

Not described

Neutrophils: seg-

Hypersegmentation

forms $3 \%$. metamy-

Of then cutrophil
Pronounced cosi-

Propounced cosi-

clocytes $1 \%$. Eosi-

nophils: scgmented

$13 \%$, myelocytes

alpha and alp

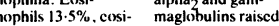

Dircet Coombs tes

Dre. Albumin re-
duced. Alpha and

duccd. Alpha 1 and
alpha and gam-
maglobulins raised

20\% \% Mature cosi-

$\begin{array}{ll}\text { Monocytes } 2 \% & \begin{array}{l}\text { nulation. No cvi- } \\ \text { dencec of leukacmia }\end{array}\end{array}$

$10 \cdot 5$ months

Initially thought to

he rhcumatic cever:

(salicylamide +

cthenazamidc), and
Nivanol (a hydan-

toin) with somc im

provement. Later:

(a)

cosinophilia

provtisonctits (5 days)
corter

Splenomegaly. Post- $\quad 188.5$

111.2

120

$-$

Incrcasc in cosinophils at myclocytc. metamyclocytc. and segmented
cells level. Slight

cells level. She
increase in
plasma cells

Albumin and
globulin normal

Eosinophilic leukacmıa

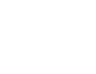

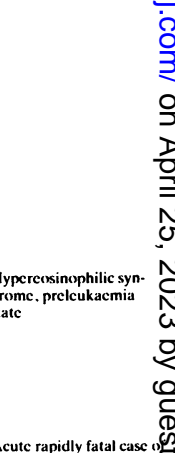

$8 \%$ Scgmented
neutrophis. $1 \%$
neutrophil band
forms. $5 \%$ lympho-
cytes. $83 \%$ cosi-

Myclocyte: Erythro- Lupus crythemato- 25 days
cytc ratio $=20.1$

Predominant cell

werc cosinoph

myclocytes.

nophils. $2 \%$ cosi-

mophil band for

metamyclicytes.
and mature cosi-

and maturc cosi-
nophils. Posimor-

tem examination:

Hypcrplasia of coss-

Hyperplasia of cosi-
nophil scries with

serum protein. pro-

tcin clce.
trophorcsis, and
immunoglobulin

immunoglobul

myclocytes

orderly maturation

Continued 
608 Alfaham, Ferguson, Sihra, and Davies

Table 2 Summary of clinical, biopsy, and necropsy findings of 19 children with the idiopathic hypereosinophilic syndrome (continued)

\begin{tabular}{|c|c|c|c|c|c|c|c|c|c|c|}
\hline Author (years) & $\begin{array}{l}\text { Age at } \\
\text { onset } \\
\text { (years) }\end{array}$ & Sex & Presenting symptoms & $\begin{array}{l}\text { Cardiovascular } \\
\text { system }\end{array}$ & Nervous system & Skin/muscle joints & Renal & Pulmonary & Gastrointestinal tract & Hepatic \\
\hline Spry $(1983)^{10}$ & 14 & $M$ & $\begin{array}{l}\text { Diarrhoea, fever, } \\
\text { sweating, abdominal } \\
\text { pain }\end{array}$ & Nonc & None & None & None & Nonc & $\begin{array}{l}\text { Diarrhoca, abdomin- } \\
\text { al pain. Biopsy: In- } \\
\text { flammatory ccll infil- } \\
\text { tration, containing } \\
\text { many cosinophils in } \\
\text { caccum. large bowcl. } \\
\text { and rectum }\end{array}$ & None \\
\hline Tallgren $(1974)^{26}$ & 3 & $\mathbf{M}$ & $\begin{array}{l}\text { Persistent febrile up- } \\
\text { per respiratory tract } \\
\text { infection, dry cough. } \\
\text { dyspnoea, arthralgia, } \\
\text { and slight knees } \\
\text { swelling }\end{array}$ & $\begin{array}{l}\text { Postmonem examina- } \\
\text { tion: Cardiomegaly, } \\
\text { pericardial cffusion, } \\
\text { epicardial petechiac. } \\
\text { Haematoma in right } \\
\text { atrial wall, white mili- } \\
\text { ary myocardial infil- } \\
\text { trate. Leukaemic in- } \\
\text { filtrate and atypical } \\
\text { cosinophils in } \\
\text { myocardium }\end{array}$ & $\begin{array}{l}\text { Postmortem examina- } \\
\text { tion: Brain swelling. } \\
\text { Lcukacmic infiltratc } \\
\text { and atypical cosi- } \\
\text { nophils in lep- } \\
\text { tomeninges }\end{array}$ & $\begin{array}{l}\text { Macular and petec- } \\
\text { hial rash. Arthralgia, } \\
\text { slight swelling of } \\
\text { knees }\end{array}$ & $\begin{array}{l}\text { Postmortem ex- } \\
\text { amination: Enlarged } \\
\text { kidncys. Leukacmic } \\
\text { infiltrate and atypical } \\
\text { eosinophils }\end{array}$ & $\begin{array}{l}\text { Dyspnoea, cough. } \\
\text { Chess x ray: Miliary } \\
\text { infiltrates. Postmor- } \\
\text { tem examination: } \\
\text { Lungs enlarged. } \\
\text { Leukacmic infiltrate } \\
\text { and atypical cosi- } \\
\text { nophils }\end{array}$ & Blceding & $\begin{array}{l}\text { Hepatomegaly. } 1, s ! \\
\text { mortem examination: } \\
\text { Liver enlargement. } \\
\text { Leukacmia infiltrate } \\
\text { and atypical eosinophi }\end{array}$ \\
\hline Thomsen $(1939)^{27}$ & 11 & $\mathbf{M}$ & $\begin{array}{l}\text { Sore throat, cervical } \\
\text { lymphadenopathy, } \\
\text { fever }\end{array}$ & $\begin{array}{l}\text { Congestive heart fai- } \\
\text { lure, cardiomegaly. } \\
\text { pericarditis. Postmor- } \\
\text { tem examination: } \\
\text { Pericardial thickening } \\
\text { and petechiac. Heart } \\
\text { dilated, right half } \\
\text { hypertrophic. Right } \\
\text { auricle cnlarged, with } \\
\text { a parietal thrombus. } \\
\text { Myocardial and en- } \\
\text { docardial fibrosis }\end{array}$ & None & $\begin{array}{l}\text { Generalised oedema. } \\
\text { especially of face. } \\
\text { Migratory polyarthri- } \\
\text { tis and joint deformi- } \\
\text { ties }\end{array}$ & $\begin{array}{l}\text { Postmonem ex- } \\
\text { amination: Enlarged } \\
\text { kidneys }\end{array}$ & $\begin{array}{l}\text { Posimortem ex- } \\
\text { amination: Oedema } \\
\text { and blast cells infil- } \\
\text { trate }\end{array}$ & None & $\begin{array}{l}\text { Hepatomegaly. Post- } \\
\text { mortem examination: } \\
\text { Blast cells infiltration }\end{array}$ \\
\hline Yam (1972) ${ }^{28}$ & 12 & $\mathbf{M}$ & $\begin{array}{l}\text { Cough, rhinorhoea. } \\
\text { fever, chest pain, } \\
\text { tingling, and numb- } \\
\text { ness of hands }\end{array}$ & $\begin{array}{l}\text { Congestive heart fai- } \\
\text { lure, cardiomegaly. } \\
\text { Posimortem examina- } \\
\text { tion: Heart dilated. } \\
\text { Mural thrombosis and } \\
\text { fibroplastic parictal } \\
\text { endocarditis }\end{array}$ & $\begin{array}{l}\text { Tingling and numb- } \\
\text { ness of hands. Hyper- } \\
\text { reflexia, clonus, and } \\
\text { bilateral Babinski } \\
\text { syndrome }\end{array}$ & $\begin{array}{l}\text { Petechiac. Erythe- } \\
\text { matous rash. Muscle } \\
\text { weakness }\end{array}$ & None & $\begin{array}{l}\text { Cough. chest pain. } \\
\text { Chest x ray: Diffuse } \\
\text { granular infiltratc } \\
\text { and enlarged hilar } \\
\text { lymph nodc. Post- } \\
\text { mortem examination: } \\
\text { Multiple infarctions } \\
\text { and diffusc pneumo- } \\
\text { nia }\end{array}$ & $\begin{array}{l}\text { Diarrhoca, vomiting, } \\
\text { nausea, abdominal } \\
\text { pain. Perforated } \\
\text { antral ulcer }\end{array}$ & $\begin{array}{l}\text { Hepatomegaly. Post- } \\
\text { mortem examination: } \\
\text { Massive liver enlarge- } \\
\text { ment. Heavy infiltrate } \\
\text { of immature eosinoph } \\
\text { and blast forms }\end{array}$ \\
\hline Present case & 14 & $\mathbf{F}$ & $\begin{array}{l}\text { Weight loss, anacmia, } \\
\text { breathlessness, } \\
\text { anorexia, Icthargy. } \\
\text { nausea, diarrhoea } \\
\text { and vomiting. ame- } \\
\text { norrhoea, petechial } \\
\text { rash, mouth ulcers. } \\
\text { blisters of hands and } \\
\text { feet, with joint and } \\
\text { limb pain }\end{array}$ & $\begin{array}{l}\text { Congestive heart fai- } \\
\text { lurc, cardiomegaly, } \\
\text { hypertension, systolic } \\
\text { murmur (mitral re- } \\
\text { gurgitation), pericar- } \\
\text { dial effusion }\end{array}$ & None & $\begin{array}{l}\text { Petcchial rash, joint } \\
\text { and limb pains }\end{array}$ & Haematuria & $\begin{array}{l}\text { Cough, breathless- } \\
\text { ness Chest x ray: In- } \\
\text { terstital lung oedc- } \\
\text { ma. vascular redis- } \\
\text { tribution. small right } \\
\text { sided pleural effusion }\end{array}$ & $\begin{array}{l}\text { Diarrhoca and } \\
\text { vomiting }\end{array}$ & Hepatomegaly \\
\hline
\end{tabular}


The idiopathic hypereosinophilic syndrome

\begin{tabular}{ll}
\hline Lymphatic/spleen & $\begin{array}{l}\text { Total white blogd } \\
\text { call count }\left(\times 10^{9} / l\right) \\
\text { at presentation }\end{array}$ \\
\hline None & 27
\end{tabular}

\begin{tabular}{l}
$\begin{array}{l}\text { Eosingphil count } \\
\left(\times 10^{9} /\right) \text { at } \\
\text { presentation }\end{array}$ \\
\hline 14.63
\end{tabular}

Haemoglobin
Haemoglohin
(g/dl) at presentation

$\begin{array}{ll}\begin{array}{l}\text { Plateless }\left(\times 10^{9} / l\right) \\ \text { at presentation }\end{array} & \begin{array}{c}\text { Peri } \\ \text { Not described }\end{array} \\ & \end{array}$

\begin{tabular}{lll}
$\begin{array}{l}\text { Peripheral blood } \\
\text { white blood cell } \\
\text { morphology }\end{array}$ & Bone marrow & $\begin{array}{l}\text { Other lahoratory } \\
\text { findings }\end{array}$ \\
\hline $\begin{array}{l}\text { Vacuolated and } \\
\text { degranulated } \\
\text { cosinophils }\end{array}$ & Not described & $\begin{array}{l}\text { Iron deficiency } \\
\text { anacmia. High } \\
\text { serum IgG }\end{array}$ \\
\hline
\end{tabular}

72. $5 \%$ Segmented eosinophils. $2 \%$ cosinophil myelo. cytes and metamyand splenomegaly. Posimoriem exmode and spleen; leukaemic infiltrate and atypical cosi-

\section{9}

$57 \cdot 275$

Normal

Normal

$31 \cdot 08$

$10 \cdot 3$

140

phadenopathy. Sple-

nomegaly. Postmor-

tem examination:

Blast cells infiltratio

of lymph

42

31.08

(a)

Initially: Increase in myeloid cells prenophils at various stages of maturation. Paralcucoblasts' were scen. Later: Myelocy with few 'paraated

Eosinophils showed Initially: Incrcasc in slightly basophilic cytoplasm, contain -
ing variable, mostly few coarse granules. few coarse granules.

appeared in increas

stem cells and cells

of eosinophil serics. made $43 \%$ and 'erythropoiesis com-
pletely paralysed' raised. Antinuclear

13.4

$5 \cdot 2$

$9 \cdot 3$

315
Mainly maturc cosinophils of differcn size, vacuolated cyenly distributed

granules, decreased

number of granules

and mixed baso-

cosinophilic gra-
nules. Nuclear

hypo- and mainly

hypersegmentation

Hypercellular. Coombs test - ve. and cosinophilia

Coombs test $-v e$. sis cell -ve.

4 months siscll

Eosinophils, show-
ing partial degraing partial
nulation
Hypercellular. Pro-
nounced eosinophilia
IgE, rheumatoid factor, alpha2 glo-

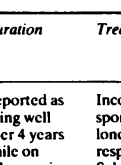

while on

Salazopyrinc.

Diarrhoea re-

turns within

one day of
stopping it

Mycoplasma orale type I isolated from bone marrow. Cold Cryoprecipitate +ve. IgM G and $A$ factor - ve.

Rheumatoid factor -ve. later became
+ve. Lupus crythematosis cell -ve.

11 months

Reduced serum albumin. Wassermann reaction and

12 months

Treatment Authors' diagnosis

Authors' diagnosis ज्ञ

Incomplete re- Hypereosinophilic syn-

ponse to predniso- drome with isolated gaș

troin trointestinal tract involve:
ment

$\frac{\text { के }}{\overparen{D}}$

$\frac{\text { จ }}{2}$

o

6-Mercaptopurine, Eosinophilic leukaemia $\overrightarrow{0}$ methotrexatc, vin- cause, result or fortuitoin cristine

cause, result or fortuitaus

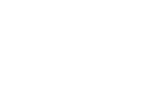

Splenic radiation and bone radiation

:

\&

Prednisolone, busulphan

Eosinophilic lcukaemia bulin and IgG raised. Albumin
low. Antinuclear factor-ve. Serum folatc low. Serum vorma!
After 15

months doing
well nate days. Eosinophils raised when prednisolone was
stopped 
cells and basophils, peptides are released that are strongly chemotactic for eosinophils, ${ }^{812}$ such as eosinophil chemotactic factor of anaphylaxis. Histamine has also been shown to be chemotactic for eosinophils. As IgE is directly involved in the triggering of mast cells with subsequent degranulation the association of eosinophilia with IgE mediated immediate hypersensitivity reaction can be explained by such chemotactic mechanisms. Eosinophil chemotactic factors that are derived from the complement system include the proteolytic fragment of C5, C5a, and the trimolecular complex C567. Other recognised factors are leucotriene B4 and substances produced by some parasites. ${ }^{12}$

On the other hand, factors that have an eosinopenic effect on blood concentrations include corticosteroids and beta adrenergic agonists, as well as an eosinopenic factor present in inflammatory exudate, which may account for the well documented eosinopenic response that is associated with most bacterial and viral infections, apart from a few exceptions (see Table 1). ${ }^{1}{ }^{12}$

Mechanisms of tissue damage in the idiopathic hypereosinophilic syndrome. In addition to their possible beneficial role, there is evidence that the eosinophils may occasionally be harmful to the host. For example, in asthma, it was generally held until recently that the eosinophils were protective, destroying the mast cell products that induce such clinical features of asthma as bronchoconstriction and changes in blood supply to the lungs. Several groups now feel, however, that the eosinophils may be responsible for some of the serious complications of asthma, including bronchial epithelial cell injury and even pulmonary fibrosis. It is the proteins in the distinctive cytoplasmic granules of the eosinophil that are suspected of damaging the lungs.'

The cytotoxic effect of eosinophil granular basic proteins on parasites has led to an examination of the potential cytotoxicity for mammalian cells. ${ }^{12}$ Cultured intestinal, splenic, cutaneous, and peripheral blood mononuclear cells are damaged by major basic proteins. There is evidence that these enzymes play a major role in the pathogenesis of the idiopathic hypereosinophilic syndrome. ${ }^{8}$ Of these proteins, the eosinophilic major basic protein, as well as the eosinophilic cationic protein, were found to be raised in the serum samples of patients with this syndrome. ${ }^{810}$ Furthermore, degranulated eosinophils in peripheral blood smears are common in idiopathic hypereosinophilic syndrome and their presence seems to be a valuable indication of endocardial disease. ${ }^{13}$ In vitro experiments with rat myocardial cells support the idea that components of the granules are released from the circulating eosinophil and directly damage the myocardium. ${ }^{14}$ Eosinophilic cationic protein may also be partially responsible for the thromboembolic phenomena characteristic of this syndrome. Eosinophil granules contain potent neurotoxin, which causes demyelination and damage Perkinje cells in laboratory animals. It might be that the same, or similar, neurotoxin causes disease in patients with this syndrome. ${ }^{12}$

Other potential mechanisms of tissue damage include tissue infiltration with eosinophils (space occupying) and thromboembolic phenomena related to thrombi associated with endomyocardial disease.

\section{Incidence}

Idiopathic hypereosinophilic syndrome mainly affects patients between 20 and 50 years of age. The incidence is not known, but over 150 patients from different age groups have now been described. After a comprehensive search of the published reports 18 cases of this syndrome were identified in children under 16 years. ${ }^{4} 71015-28$

Summary of the clinical details and laboratory, biopsy, and autopsy findings of the involvement of the various organ systems of the 18 children reported in these reports and the present case is shown in Table 2. Fourteen boys and five girls were affected-that is, the male predominance is less pronounced than in older age groups.

\section{The cardiovascular system in the idiopathic hypereosinophilic syndrome}

Involvement of the cardiovascular system is the main source of morbidity and mortality. Sixteen children showed evidence of this. Cardiovascular involvement in these children was generally similar to that in adult patients in its frequency and distribution.

The cardiovascular involvement in this syndrome is nearly always biventricular, but it spares the outflow tracts. Characteristically, patients develop endocarditis with overlying thrombosis and, later, endomyocardial fibrosis. Involvement of the supporting structures of the atrioventricular valves often results in mitral and sometimes tricuspid regurgitation. The endocardial thrombi give rise to peripheral emboli, which further complicate the clinical picture. Patients with this syndrome are also at increased risk of developing thromboembolic disease in blood vessels of medium to large size. Disease in small blood vessels also occurs, with thickening of intimal tissue. ${ }^{6}$

Cases that were previously called Loeffler's eosinophilic endocarditis are probably synonymous with hypereosinophilic syndrome with severe cardiac involvement. It is now accepted that there is a range of eosinophilic heart disease, varying in severity 
from Loeffler's eosinophilic endocarditis, the most acute and fatal form, to Ugandan type endomyocardial fibrosis. The heart in the condition described in 1948 in West Africa by Davies, originally called endocardial fibrosis and later endomyocardial fibrosis, ${ }^{29}$ is indistinguishable from the heart of a patient with Loeffler's fibroplastic parietal endocarditis, or eosinophilic leukaemia. Further, endomyocardial fibrosis is also known to present in non-tropical countries.

There have been reports of patients who present with an initial hypereosinophilic illness, survive, and are found at necropsy to have endomyocardial fibrosis, sometimes with features identical to Ugandan type endomyocardial fibrosis. ${ }^{30}$ Endomyocardial fibrosis seems to be the burnt out phase of an eosinophilic heart disease.

Hearts from patients with heart failure secondary to other causes of hypereosinophilia also had necropsy appearances similar to those of patients with the idiopathic hypereosinophilic syndrome. Thus the common denominator of the cardiac lesion is probably the eosinophilia itself and not an unrecognised underlying factor. ${ }^{8}$

In a study of 50 patients Harley et al defined the non-cardiovascular characteristics that distinguish patients at risk of developing endomyocardial fibrosis from those who remained free of heart disease. ${ }^{31}$ Patients with clinically overt heart disease were more likely $(p<0.05)$ to be male and HLA-Bw44 positive and have splenomegaly, thrombocytopenia, raised serum vitamin $B_{12}$ concentrations, and hypogranulated or vacuolated eosinophils and abnormal early myeloid precursors in the peripheral blood. Those patients with heart disease were also more likely to have fibrosis and decreased megakaryocytes in the bone marrow. In contrast, those who remained free of heart disease tended to be female and have angio-oedema, hypergammaglobulinaemia, raised serum IgE concentration, and circulating immune complexes. Appreciation of this relative degree of risk for the major complication of the idiopathic hypereosinophilic syndrome should prove useful in the early identification and appropriate treatment of patients in whom endomyocardial fibrosis might develop.

\section{Involvement of other organs/systems}

The clinical manifestation and pathological findings of the involvement of the various systems in those children who had cardiovascular involvement might have been secondary to the latter either in the form of congestive heart failure, causing, for example, pulmonary or gastrointestinal symptoms or hepatomegaly, or embolic phenomenon, originating from intracardiac thrombosis. In many cases, however, the primary involvement of the various systems was also proved by biopsy or necropsy findings (Table 2).

In addition to the involvement of the cardiovascular system, which was the major problem in our present case, there are certain other features that need some emphasis.

(1) Previous history of asthma. None of the other 18 children had a similar history. Also there are a few well described adult patients who had both bronchial asthma and this syndrome. ${ }^{6} 10$

(2) Dramatic response to corticosteroids.

(3) Raised serum rheumatoid factor. This has rarely been observed in patients with this syndrome. ${ }^{9} 32$ Furthermore, the concentration decreased after treatment.

(4) The serum IgE concentration returned to normal after treatment. This has also been reported by Bush. ${ }^{32}$ The changes in serum IgE concentration might be of benefit in monitoring progress, as is the eosinophil count.

(5) Low total white blood cell count at presentation, which, like initial high serum IgE concentration, has been reported to be associated with a better response to treatment.

(6) In our case, her illness initially started with mouth ulcers and blisters of hands and feet. This combination has not been reported before. Presentation of the idiopathic hypereosinophilic syndrome with mouth ulcers has been reported in two patients, who were 26 and 51 years old. ${ }^{33}$ In these patients, however, there was ulceration of other mucous membranes, including the genitalia.

\section{Treatment}

The idiopathic hypereosinophilic syndrome is a rare disorder, but one that is accompanied by high morbidity and mortality. Recent vigorous treatment regimens, however, directed at lowering the eosinophil count with symptomatic treatment of complications of the organ systems, has brought about a tremendous improvement in survival and prognosis. The mainstay of treatment is corticosteroids. Hydroxyurea is used in patients who do not respond or only partially respond to steroids..$^{34}$ Its use may also be required to allow the steroid dosages to be reduced in patients who require maintenance treatment. Vincristine has also been used recently in a number of patients with aggressive disease. Anticoagulants are required in most patients. ${ }^{10}$ Drugs that inhibit platelet function-for example, dipyridamole, have also been used. Leucophoresis and plasma exchange do not seem to have any clinical 
benefit, unless the patient also had increased whole blood viscosity and widespread vessel occlusions.

Patients without dysfunction of organ systems are not treated and are followed closely at three to six monthly intervals. ${ }^{34}$ If patients show dysfunction of organ systems they are treated initially with corticosteroids. If the disease stabilises or improves the steroid is continued on an alternate day regimen. Ultimately, it is tapered to the lowest possible dose that will control that disease. If the disease progresses despite steroids a cytotoxic agent, specifically hydroxyurea, is added with the aim of maintaining the leucocyte count at less than $10 \times 10^{9} / 1$ with a normal eosinophil count.

\section{Prognosis}

Favourable prognostic factors that indicate better response to treatment are high serum IgE concentration, the presence of angioneurotic oedema, and unmistakable eosinopenia within a few hours after the first dose of prednisolone. Unfavourable prognostic signs are pronounced leucocytosis and myeloblasts in peripheral blood and congestive heart failure. ${ }^{434}$ It is also important to look for the possibility of high serum vitamin $B_{12}$ and low serum folate concentrations, abnormal leucocyte alkaline phosphatase activity, chromosomal abnormalities, and basophilia of more than $3 \%$. These leukaemic markers as a group seem to have a predictive value in terms of symptomatology and response to treatment. Those with positive findings had more severe disease. $^{4}$

\section{Eosinophilic leukaemia}

Many patients of various age groups with this syndrome, including eight children in our review, were reported as having eosinophilic leukaemia. Although this is one extreme of this syndrome, most patients do not seem to have leukaemia. Rickles and Miller suggested the following criteria for a diagnosis of eosinophilic leukaemia. ${ }^{35}$

(1) Pronounced and persistent eosinophilia, associated with immature forms, either in the peripheral blood or bone marrow.

(2) Greater than $5 \%$ blast forms in the bone marrow.

(3) Tissue infiltration by immature cells of predominantly eosinophilic type.

(4) An acute natural history measured in months, accompanied by anaemia, thrombocytopenia, increased susceptibility to infection, and/or haemorrhage.

Although the hypereosinophilic syndrome was originally defined in the context of primary eosinophilia, it is now recognised that the same involvement of organ systems may arise when eosinophilia occurs secondary to a defined condition-for example, drug reactions and parasitic disease ${ }^{124}$ -so that early identification and prompt treatment of the primary condition, which usually results in the disappearance of the hypereosinophilia, may prevent these complications.

We thank Professor C J F Spry for reading the paper and for his helpful advice, Mrs T Wisniewski for technical help, Mr G Titley for his help in the search of the publications, and the Medical Illustration Department at the Royal Gwent Hospital.

\section{References}

${ }^{1}$ Spry CJF. Eosinophilia. Practitioner 1982;226:79-88.

2 Lukens JN. Eosinophilia in children. Pediatr Clin North Am 1972;19:969-81.

${ }^{3}$ Hardy WR, Anderson RE. The hypereosinophilic syndromes. Ann Intern Med 1968;68:1220-9.

${ }^{4}$ Chusid MJ, Dale DC, West BC, Wolff SM. The hypereosinophilic syndrome: analysis of fourteen cases with review of the literature. Medicine (Baltimore) 1975;54:1-27.

5 Trounce JQ, Tanner MS. Eosinophilic gastroenteritis. Arch Dis Child 1985;60:1186-8.

${ }^{6}$ Spry CJF. The hypereosinophilic syndrome: clinical features, laboratory findings and treatment. Allergy 1982;37:539-51.

${ }^{7}$ Engfeldt B, Zetterstrom R. Disseminated eosinophilic "collagen disease": a clinical and pathological study of a clinical entity related to Loeffler's syndromes. Acta Med Scand 1956;153: 337-53.

${ }^{8}$ Fauci AS, Harley JB, Roberts WC, Ferrans VJ, Gralnick HR, Bjornson $\mathrm{BH}$. The idiopathic hypereosinophilic syndrome: clinical, pathophysiologic and therapeutic considerations. Ann Intern Med 1982;97:78-92.

${ }^{9}$ Parrillo JE, Lawley TJ, Frank MM, Kaplan AP, Fauci AS. Immunologic reactivity in hypereosinophilic syndrome. J Allergy Clin Immunol 1979;64:113-21.

10 Spry CJF, Davies J, Tai PC, Olsen EGJ, Oakley CM, Goodwin JF. Clinical features of fifteen patients with the hypereosinophilic syndrome. $Q J$ Med 1983;52:1-22.

11 Carey JP, Burke AC, Carter WH. Transient hypereosinophilia in the infant of a mother with hypereosinophilic syndrome. Arch Intern Med 1982;142:1754-5.

12 Weller PF. Eosinophilia. J Allergy Clin Immunol 1984;73:1-10.

13 Olsen EG, Spry CJF. The pathogenesis of Löfler's endomyocardial disease and its relationship to endomyocardial fibrosis. Prog Cardiol 1979;8:281-303.

14 Tai PC, Hayes DJ, Clark JB, Spry CJF. Toxic effects of eosinophil secretion products on isolated rat heart cells in vitro. Biochem J 1982;204:75-80.

15 Bentley HP, Reardon AE, Knoedler JP, Krivit W. Eosinophilic leukemia: report of a case, with review and classification. Am J Med 1961;30:310-22.

${ }^{16}$ De Vaan GAM, van Haelst UJG. Hypereosinophilia syndrome or eosinophilic leukaemia: a case report of a one year old infant. Helv Paediatr Acta 1979;34:271-9.

17 Donohue WL, Snelling CE, Jackson SH, et al. Pituitary adrenocorticotropic hormone (ACTH) therapy in eosinophilic leukaemia. JAMA 1950;143:154-7.

${ }^{18}$ Engbaek HC, Heerup L, Thomsen S. Om den eosinofile leukaemi og "eosinophilia Leukaemoides". Nord Med 1942;14:1535-51.

${ }^{19}$ Foasso M-F, Hermier M, Age C, Mermillon M, Francois R. Le syndrome hypereosinophilique avec atteinte endomyocardique chez l'enfant; analyse critique de la litterature a propos d'un cas. Pediatrie 1984;39:147-55. 
20) Goldstein von R. Eosinophilia infectiosa. Annales Paediatrici 1952;179:216-26.

${ }^{21}$ Herrera-Goepfert R, Larraza-Hernandez O. Sindrome hipereosinofilico. Bol Med Hosp Infant Mex 1985;42:201-6.

${ }^{22}$ Knorr D, Scheppe KJ. Endocarditis parietalis fibroplastica mit Bluteosinophilie (Loffler) bei einem siebenjahrigen Kind. Zeits chift fur Kinderheilkunde 1958;81:102-12.

${ }^{23}$ Libanoff AJ, McMahon NJ, Eosinophilia and endomyocardial fibrosis. Am J Cardiol 1976;37:438-41.

24 Olson TA, Virmani R, Ansinelli RA, et al. Cardiomyopathy in a child with hypereosinophilic syndrome. Pediatr Cardiol 1982;3:161-9.

${ }^{25}$ Rasche RFH, Kelsch RD, Weaver DK. Löffler's endocarditis in childhood. Br Heart J 1973;35:774-6.

${ }^{26}$ Tallgren LG, Wegelius R, Andersson LC, Jansson E. Eosinophilic leukaemia: recovery of mycoplasma orale from the bone marrow. Acta Med Scand 1974;195:87-92.

27 Thomsen S, Plum P. Eosinophilic leukemia. Acta Med Scand 1939;101:116-37.

${ }^{28}$ Yam LT, Li Cy, Necheles RF, Katayama I. Pseudoeosinophilia, eosinophilic endocarditis and eosinophilic leukaemia. Am J Med 1972;53:193.

29 Davies .JNP. Endocardial fibrosis in Africans. East Afr Med J 1948;25:10-4.

30) Chew CYC, Ziady GM, Raphael MJ, Nellen M, Oakley CM.
Primary restrictive cardiomyopathy:non-tropical endomyocardial fibrosis and hypereosinophilic heart disease. Br Heart $J$ 1977; 39:399-413.

${ }^{31}$ Harley JB, Fauci AS, Gralnick HR. Noncardiovascular findings associated with heart disease in idiopathic hypereosinophilic syndrome. Am J Cardiol 1983;52:321-4.

32 Bush RK, Geller M, Busse WW, Flaherty DK, Dickie HA Response to corticosteroids in hypereosinophilic syndrome: association with increased serum IgE levels. Arch Intern Med 1978; 138:1244-6.

${ }^{33}$ Leiferman KM, O'Duffy JD, Perry HO, et al. Recurrent incapacitating mucosal ulcerations. A prodrome of the hypereosinophilic syndrome. JAMA 1982;247:1018-20.

${ }^{34}$ Parrillo JE, Fauci AS, Wolff SM. The hypereosinophilic syndrome: dramatic response to therapeutic intervention. Trans Assoc Am Physicians 1977;90:135-44.

35 Rickles FR, Miller DR. Eosinophilic leukemoid reaction: report of a case, its relationship to eosinophilic leukemia and review of the pediatric literature. J Pediatr 1972;80:418-28.

Correspondence to Dr M A Alfaham. Department of Child Health, University Hospital of Wales, Heath Park, Cardiff CF4 $4 \mathrm{XN}$, Wales.

Received 5 January 1987 